\title{
Smart Car Automated System to Assist the Driverin Detecting the Problem and Providing the Solution
}

\author{
S.Veena, K.Ramyadevi, K.Elavarasi, M.Preetha
}

\begin{abstract}
Intodays world, Mobile application plays a very important role to process any type of data in hand. The mobile application is mainly developed for the drivers to work in mobile devices and to run on tablets and smart phones. These applications are used for various purposes like web browsing, calendar, buying music, gaming, traveling, entertainment, social networking and productivity. The mobile applications differ from the integrated software systems found in Personal Computers by its isolated functionality. The motor Car belongs to the mechanical world, and the functionality is not aware by all the drivers, thus it becomes a difficult one to handle it. In the existing system, there is no diagnostic system launched in mobile application for the drivers to find the minor problems in each and every car. There is only a manual book given to the drivers for motorcar by the manufacturer to see how to start a car and the parts of the car are given in detail. There is nothingmentioned about the minor problems that happens in the motorcar and the solution to recover it.To overcome the problems faced by the Drivers on the road and to help them in emergency situation, a Self Detection and recovery system is proposed for the Drivers, especially for the Learning drivers to detect the minorproblems in the vehicles and also to recover from those problems by using this automized smart system. Through this smart mobile application, the driver can find solutions for all the minor problems faced in the motorcar. The driver will be aware of exact fault and the driver can take necessary action accordingly. The proposed system acts as a good driver friendly interface for any Driver to detect the minor problem easily by using the alert signals and also to recover from these problems easily by searching through the terms, which usually denote the type of the problem and it accordingly find out the solutions, which would be highly helpful to proceed without interruption. The proposed system a low cost application wherein the driver has to sign up and find the solutions to the problems. This mobile application is secured because only the authorized driver can login and see the solutions but not by anyone else. This application gives the correct and exact solution to all the minor problems encountered by the mobile driver.
\end{abstract}

Keywords: Android Operating Systems, Detection system, Driver assistance, Intelligent Vehicles, Mobile application, Vehicle safety.

Revised Manuscript Received on April 12, 2019.

S.Veena, Professor, Department of Computer Science and Engineering,S.A Engineering College, Chennai-77.T.N, India. (veenas@saec.ac.in)

K.Ramyadevi, Assistant Professor, Department of Computer Science and Engineering,S.A Engineering College, Chennai-77.T.N, India.(ramyadevik@saec.ac.in)

K.Elavarasi,Assistant Professor, Department of Computer Science and Engineering,S.A Engineering College, Chennai-77.T.N India.(elavarasi@saaec.ac.in)

M.Preetha,Department of Computer Science and Engineering,S.A Engineering College, Chennai-77.T.N, India. (drpreetha @ saec.ac.in)

\section{INTRODUCTION}

In today's world, as technology constantly changing, the number of drivers has been increased and many drivers have been attached to their mobile devices. In an age when smart phones and tablets have become the one-stop-shop for communication andentertainment, people are also increasingly using them to accomplish work-related tasks with a simple swipe of the finger.

A mobile application, most commonly referred to as an app, is a type of application software, which is designed to run on a mobile device, such as a smart phone or tablet computer. Mobile applications frequently serve to provide drivers with similar services to those accessed on Personal Computers. The mobile applications are generally small and individual software units but with limited functionality. This use of software has been popularized by Apple Inc. and its App Store, which sells thousands of applications for the iPhone, iPad and iPod Touch.

\section{Types of Mobile Application}

Native Application has been developed for use on a particular platform or device. A native mobile application is also known as Smartphone application, which is coded in programming languages like Java for Android operating systems and Objective $\mathrm{C}$ for iOS. These applications provide very fast performance and also gives a high degree of reliability. Also, theseapplications have access to various devices of a phone like address book and camera. In these applications, the drivers can use few applications without an Internet connection.

Hybrid Applications are similar to native applications, which are written with web technologies like CSS, HTML5 and JavaScript and run on the device. These applications run inside a native container, and leverage the device's browser engine to render the HTML and process the JavaScript locally. A layer namely web-to-native abstraction, which enables access to devicecapabilities that are not accessible in Mobile Webapplications, like an accelerometer, camera and local storage.

Web Application are usually stored on a remote server, which are delivered over the internet through a browser. Web applications are not called as real applications, but they are really websites, which look and feel like native applications. They are written in HTML5 and run by a 


\section{Smart Car Automated System To Assist The Driver In Detecting The Problem And Providing The Solution}

browser. Drivers access the Web application as similar toaccess any web page.

\section{Android Operating System}

Android is open source and Google releases the code under the Apache License. This has an open source code and permissive licensing, which permits the open source code to modify for free and also to be distributed by device manufacturers, wireless carriers and enthusiast developers. Android has a large community of developers writing applications that extend the functionality of devices, written primarily in a customized version of the Java programming language. These features makes Android to become the world's most widely used smart phone platform, overtaking Symbian in the fourth quarter of 2010, and the software of choice for technology companies who require a low-cost, customizable, lightweight operating system for devices without developing one from scratch. As a result, despite being primarily designed for phones and tablets, it has seen additional applications on televisions, games consoles and other electronics. Android's nature adds new features for advanced drivers or bring Android to devices which were officially released running other operating systems. The success of Android has made it a target for patent litigation as part of the so-called "smart phone wars" between technology companies.

\section{RELATED WORKS}

Akhila V Khanapuri [1], discussed about the dawn of the smartphone era, phones area unit used for much more than simply a straightforward device for communication. These phones possess process power capable of acting complex functionalities. Additionally, On Board Diagnostics (OBD II) customary that has been mandated since 1994 is finding use for in-vehicle applications because of the supply of Bluetooth OBD-II connectors. These connectors bind to the vehicle's Electronic Control Unit and change transparent property between smart phone device and vehicle's electronic control unit.

Doo Seop Yun [2], planned a vehicle includes numerous electronic devices victimization IT technologies and vehicles that have safety and convenience devices increase bit by bit. Mostly, hardware device was core in existing vehicles, however latest vehicles are focusing on package. From the results, consultants expect that importance of IT devices can increase over four-hundredth by 2015 and that they predict that role of package can grow consistently. But, vehicle info primarily based application services solely give info specialized for every vehicle manufacture and model.

Dr.SuebskulPhiphobmongkolSupakornMasiri

planned an automotive is not maintained at the desired schedule, the warrant could be void. Automotive maintenance schedule, either by distance or time will vary by models and makers. With the upper capability of mobile devices and good phones, automotive maintenance application is possible. Automation is one in every of the foremost wide used software system on mobile devices. Associate automaton devices often go with a GPS module inbuilt. Therefore it will notice location in real time. If the applying for measure the accumulated distance and pursuit location is put in on associate automaton device, it will offer data to drive the operation of the projected application. The projected application works on the mobile device or good phones with automaton operational System. The projected application provides basic data of automotive maintenance within the mobile information, so as to notify to car's driver for having maintenance on acceptable mileage or period. The projected application conjointly provides location pursuit data that keep from the movement information of automotive.

GunjanChugh [4], had proposed a technique for monitoring the road conditions. In this paper, importance of roadis highlighted andhow it plays an important part inpeople's daily lives. After construction, once the road is place into use, it causeanomalies, as a result of continuous rolling beneath the wheels, and snow, rain and alternative natural factors. This anomaly can have an effect on the standard of driving. With the provision of data concerning the road conditions, road drivers can be cautious regarding or avoid the dangerous roads. After working on observation of the road conditions, it has gained important attention in recent time. The traffic conditions in developing countries, like India, are a lot of complicated thanks to varied road conditions, heterogeneous mixture of vehicles and chaotic traffic. Since India is a developing country there could be a constant demand permanently quality transportation, infrastructure and services. Roads are required to be monitored endlessly for roughness and alternative anomalies to avoid inconvenience to the road drivers. Road observation can even facilitate to predict the estimated point from one place to a different. This paper presents an in depth survey of ways for police work road conditions.

Felipe Castillo [5], planned an automotive has positioned itself because the predominant mean of transport within the world and it has become a key instrument for the functioning of society. However, over the last decades, the planet has experienced associate degree intense method of environmental awareness, where the auto plays a big role by being one in all the main pollution sources that exist within the planet.

Jakob Eriksson [6], planned and have deployed P2 on seven taxis running within the Boston space. Employing an easy machine-learning approach, we show that we tend to area unit ready to establish potholes and different severe paved surface anomalies from measuring instrument knowledge. Via careful choice of coaching data and signal options, we've been ready to build a detector that misidentifies sensible road segments as having potholes but $0.2 \%$ of the time. We tend to judge our system on knowledge from thousands of kilometers of taxi drives, and show that it will with success sight a number of real potholes in and round the Bean Town space. After clustering to more scale back spurious detections, manual review of rumored potholes shows that over ninetieth contain road anomalies in want of repair.

Jorge Zaldivar [7], planned a widespread adoption of mobile telecommunication has remarkably improved social communications in our society. Nowadays mobile phones 
match tiny multifunction computers, being characterized by a central processing unit power and RAM size similar to that of portable computer computers offered solely a number of years ago. the longer term trend is that a lot of and a lot of drivers own these intelligent mobile terminals, which their main use bit by bit shifts towards functionalities as well as internet surf riding, social networking, transmission streaming, on-line games, domestic applications, and so on. Below these premises, it's potential to introduce novel services victimization smartphones in many various contexts.

\section{DESIGN OF ASMART CAR AUTOMATED SYSTEM FOR DRIVERS}

The proposed system also called as a Smart Car Automated System, since it detects and recovers the minor problems in a car in case of emergency. This presents a real time android application to find the minor problems that occurs in the motorcar by the driver. Through this mobile application, the driver can find solutions for the problems that are faced in the Motorcar. The Drivers or drivers need not waste money unnecessarily once if he knows the symptoms for the problems. The driver will be aware of exact fault and the driver can take necessary action accordingly. The driver can also check whether the mechanic is undertaking the right solution or not. The driver need not pay money blindly for these minor problems once if the driver know the problems and solutions to that problem. It is a low cost application wherein the driver have to sign up and find the solutions to the problems. This mobile application is secured because only the driver can login and see the solutions not by anyone else. This application gives the correct and exact solution to the problems faced by the driver.

The architecture shows the working of the Self Detection and recovery System in a Car forthe Drivers using a Smart Mobile application.

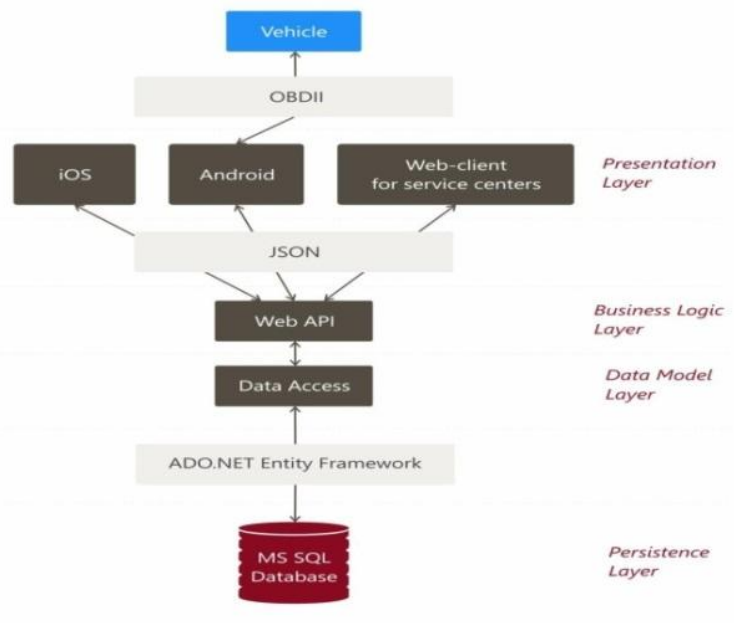
Figure 1 Architecture of a Smart
Car Automated system

The proposed system has various functionality namely Looks like, Feels like, Smells like, Sounds like and Not working.

\section{Looks Like}

In this module the driver can find the problems by their naked eye. For example if the driver finds the color of the smoke is different than the regular color means there is something wrong in the motorcar and it has to be viewed. The driver can find the solution depending on the color of the smoke the driver has observed. Same way the driver can see the smoke color, poor gas mileage, a tire wearing out, warning light on and high engine temperature through their naked eye and this module gives solutions to all the problems mentioned above.

\section{Feels Like}

In this module the driver can find solutions to the problems which the driver feels when the driver is driving the motorcar. For example problems like shaking, abnormal breaking, vehicle may be sluggish, awkward shifting, vehicle may ride uncomfortably and abnormal steering. In this module each and every solution for the mentioned problems was given correctly.

\section{Smells Like}

In this module the driver can find solutions to the problems which the driver smells when the driver drives the motorcar. For example if the driver finds rotten egg smell means the driver have to check the air filter and see whether it is dirty or not. If the air filter is dirty means the driver must replace the air filter and if the air filter is not dirty means the driver have to check the fuel injection sensor. This module gives a clear solution for the problems faced by the driver.

\section{Sounds Like}

In this module the driver can find solutions to the problems depending on what the driver hears. For example sounds like pinging, engine running roughly, engine backfires, ticking/tapping, squealing noise, sputter and cough, brakes make noise, clicking sound and clunking sound. These are the sound problems faced by the driver and this module gives solution to each and every problems.

\section{Not Working}

In this module the driver can find solutions to the problems where the motorcar didn't start and many problems like that are faced by the driver. There are a number of reasons which can cause a car engine not to start, the most common, of course, being a dead battery. Pay special attention to the noise it makes when you turn the key. Is the car completely silent? If so, there may be a problem with your battery terminal cable connections.

\section{SYSTEM PERFORMANCE \& RESULTS}

The goal of this mobile application is to find solutions to the minor problems that occurs in the motorcar, where in the driver detects the minor problems, the reason for this problem and recover from the minor problem. 


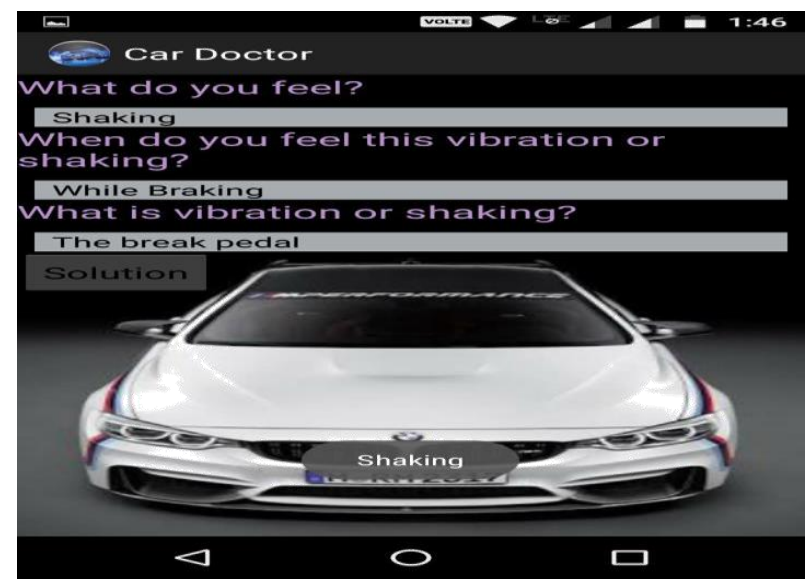

Figure 2 - Smart Car Automated System - Driver Login

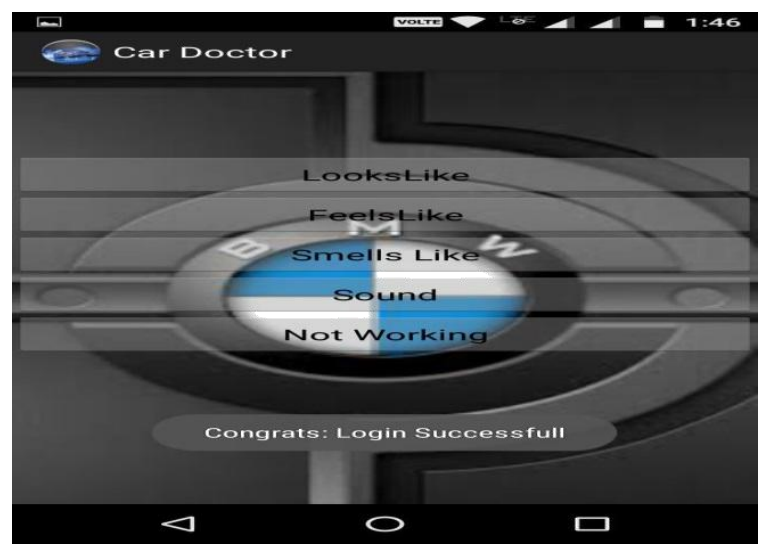

Figure 3 Smart Car Automated System - Feel

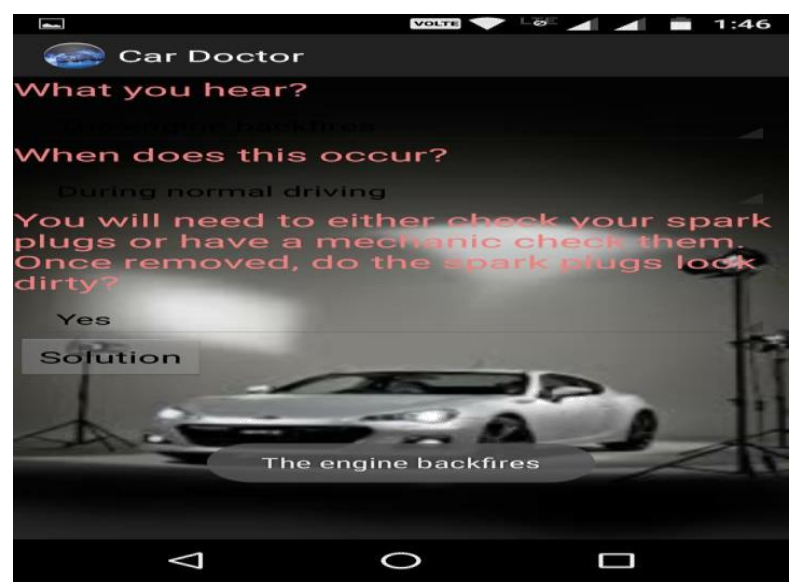

Figure 4 Smart Car Automated System - Smell

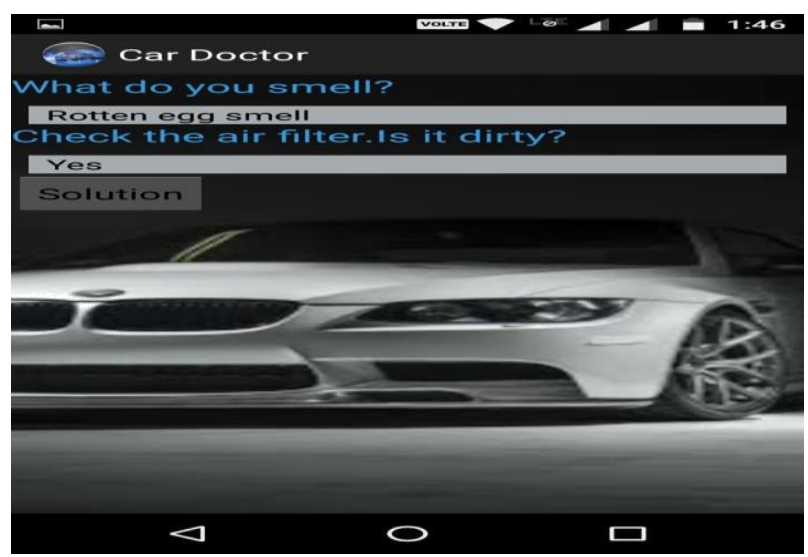

Figure 5 Smart Car Automated System- Look

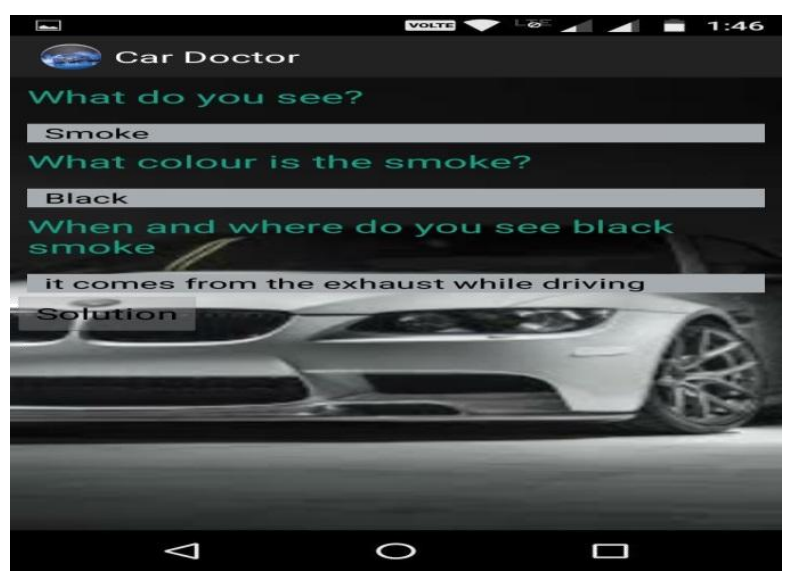

Figure 6 Smart Car Automated System -Sound

\section{CONCLUSION}

The proposed Smart Car Automated System is developed to detect and to recover the minor problems by the Drivers that occurs in the motorcar using mobile application software. The proposed system works on the mobile device or smart phones with Android Operating System. The proposed system provides basic information of car maintenance in the mobile database for the better life of the motorcar. The proposedsystem acts as a good driver friendly interface for any Driver to detect the minor problem easily by using the alert messages and also to recover from these problems easily by searching through the terms and find out the solutions, which would be highly helpful to proceed without interruption.

\section{REFERENCES}

1. Akhila V Khanapuri, AnaghaShastri, Gareth D'souza and Shannon D'souza, "On Road: A car assistant application' 2015", International Conference on Technologies for Sustainable Development (ICTSD-2015), 2015

2. Doo Seop Yun, Jeong Woo Lee, Shin Kyung Lee and OhCheon Kwon,"Development of Mobile Common Component for Providing Vehicle Information on Mobile Device", Vehicle Networking Researching Team Electronics and Telecommunication Research Institute. (ICTSD-2014), March. 05 - 07, 2014.

3. Dr.SuebskulPhiphobmongkolSupakornMasiri, "Car Maintenance Notification Application On Android Platform", International Journal of Information Technology \& Computer Science, Volume 14 Issue No 1, May 2014.

4. GunjanChugh, DivyaBansal and SanjeevSofat, "Road Condition Detection Using Smartphone Sensors:A Survey", International Journal of Electronic and Electrical Engineering, ISSN 0974-2174, Volume 7, No 6, 2014

5. Felipe Castillo, Darwin Alulema, Freddy Acosta and DerlinMorocho, "Design and Implementation of an Automatic Management System for vehicles using the Android Platform", CHILEAN Conference on Electrical, Electronics Engineering and Communication technologies, 28-30 October 2015.

6. Jakob Eriksson, Lewis Girod, Bret Hull, Ryan Newton, Samuel Madden, HariBalakrishnan, "The Pothole Patrol: Using a Mobile Sensor Network for Road Surface Monitoring", The Sixth Annual International conference on Mobile Systems, Applications and Services (MobiSys 2008), June 2008.

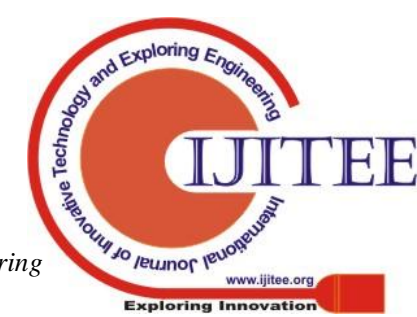


7. Jorge Zaldivar, Carlos T. Calafate, Juan Carlos Cano and Pietro Manzoni, 'Providing Accident Detection in Vehicular Networks through OBD-II Devices and Android-based Smartphones',5thIEEE $36^{\text {th }}$ Conference on Local Computer Networks, LCN, October 4 -7, 2011.

8. OnkarPathare,NavitDhande,DipakShindeand RoshanPatil, "Mobile Application for Monitoring Inefficient and Unsafe Driving Behaviour", International Journal of Advanced Research in Computer and Communication Engineering, Vol. 5, Issue 5, May 2016.

9. Sang Hyun Park and Sang Yub Lee, "Development of OnBoard Diagnosis via CAN for a HVI (Human Vehicle Interface) technology", 10th IEEE International Symposium on Parallel and Distributed Processing with Applications, 2012. 